\title{
EL IMPACTO DE EL NIÑO SOBRE LA PRODUCCIÓN DE CONCHA DE ABANICO (Argopecten purpuratus) EN BAHÍA INDEPENDENCIA, PISCO, PERÚ
}

Jaime Mendo ${ }^{1}$ y Matthias Wolff ${ }^{2}$

\section{Resumen}

La Bahía Independencia puede ser considerada a nivel mundial como una de las zonas mas productivas en invertebrados bentónicos, cuyas capturas están sujetas a fluctuaciones fuertes originadas por la ocurrencia del fenómeno El Niño y La Niña y por la falta de medidas de manejo adecuadas. Durante períodos con calentamientos fuertes los desembarques anuales alcanzan las $50000 \mathrm{t}$ en un área de aproximadamente $150 \mathrm{~km}^{2}$ y durante años fríos estas se mantienen alrededor de 500 a $1000 \mathrm{t}$.

El presente estudio analiza los cambios de los desembarques en la Bahía Independencia observados durante las dos últimas décadas y discute los principales factores que afectan los "booms" de concha de abanico. En este sentido se usa información sobre capturas, temperatura y aquella relacionada con el crecimiento, reproducción, prelación, densidad media y condiciones de oxígeno procedente de trabajos publicados y no publicados.

La relación entre la captura anual y la temperatura promedio durante el periodo reproductivo de la concha de abanico durante los últimos 20 años, muestra que la producción es afectada positivamente solo con la ocurrencia de El Niño fuertes como en 1983 y 1998.

Los resultados muestran que los "booms" de concha de abanico durante estos El Niño se debe al efecto combinado de (1) incremento en la actividad reproductiva a través de una aceleración de la maduración y un incremento de la frecuencia de desove; (2) acortamiento del periodo larval e incremento en la supervivencia larval; (3) incremento en el rendimiento en crecimiento individual; (4) incremento en la supervivencia de juveniles y adultos debido a la reducción de la biomasa de predadores; (5) incremento en la capacidad de carga de los bancos de conchas debido a elevados niveles de oxígeno.

En el marco de discusión sobre los factores que contribuyen al desarrollo de la población de concha de abanico durante el evento El Niño, no podemos dejar de considerar en detalle el impacto de la pesquería. Considerando que el volumen de captura no solo depende de la abundancia relativa de la especie, la estrategia de pesca aplicada fue analizada con el fin de explicar los diferentes niveles de producción de concha de abanico durante El Niño 1983 y 1998.

Palabras clave: El Niño, desembarques, manejo, Argopecten purpuratus, Perú

\begin{abstract}
Independencia Bay can be considered as one of the most productive invertebrate fishing grounds worldwide. One of the most important exploited species is the scallop (Argopecten purpuratus) with strong catching fluctuations related to El Niño and La Niña events and to inadequate management strategies. During strong warming periods annual landings reach up to 50 $000 \mathrm{t}$ in an area of about $150 \mathrm{~km}^{2}$ and during cold years they remain around 500 to $1000 \mathrm{t}$.

This study analyses the changes in scallop landings at Independencia Bay observed during the last two decades and discusses the main factors affecting the scallop proliferations during the El Niño events. In this way data on landings, sea surface temperature and those related to growth, reproduction, predation, mean density and oxygen concentration from published and unpublished papers are used.

The relationship between annual catches and average water temperature over the preceding reproductive period of the scallop over the past 20 year's period, showed that scallop production is affected positively only with strong El Niño such as those of 1983 and 1998.

Our review showed that the scallop stock proliferation can be traced to the combined effect of (1) an increase in reproductive output through an acceleration of gonad maturation and a higher spawning frequency; (2) a shortening of the larval period and an increase in larval survival; (3) an increase in the individual growth performance; (4) an increase in the juvenile and adult survival through reduction of predator biomass; (5) an increase in carrying capacity of the scallop banks due to elevated oxygen levels.
\end{abstract}

\footnotetext{
${ }^{1}$ Facultad de Pesquería, Universidad Nacional Agraria la Molina, Apto. 456, Lima 100, Perú Correo electrónicol: jmendo@lamolina.edu.pe

${ }^{2}$ Center for Tropical marine Ecology, ZMT, Fahrenheitstraße 1, 28359 Bremen, Alemania

e-mail:mwolff@zmt.uni-bremen.de
} 
When discussing the factors that contribute to the development of the scallop population during an El Niño event, the fishery must be considered in detail. Since the volume of the catch not only depends on the relative abundance of the species, fishing efforts and strategies were analyzed in order to explain different levels of scallop production during El Niño 1983 and 1998.

Key words: El Niño, landings, management, Argopecten purpuratus, Peru

\section{Introducción}

Las aguas de la costa peruana están consideradas como las más productivas del mundo por sus altos niveles de productividad primaria y secundaria originado por los afloramientos de aguas ricas en nutrientes hacia la superficie (Rhyther, 1969). La pesquería artesanal costera, con una producción anual de cerca de 200000 t, aparece como insignificante comparada con la bien conocida pesquería de pequeños pelágicos, la cual en algunos años supera los 10 millones de toneladas anuales, (Espino \& Wosnitza-Mendo, 1988). Sin embargo, esta pesquería que explota alrededor de 170 especies de peces y 30 especies de invertebrados de alto valor comercial (Estrella \& Guevara, 1998) da empleo a miles de pescadores y a sus familias.

La mayoría de los invertebrados bentónicos son capturados por buzos que colectan estos manualmente del fondo, algunos son desprendidos del sustrato (Concholepas, Fisurela sp.) y otros como el pulpo son extraídos con un gancho de sus refugios. El centro de la pesquería de buceo es la Bahía Independencia

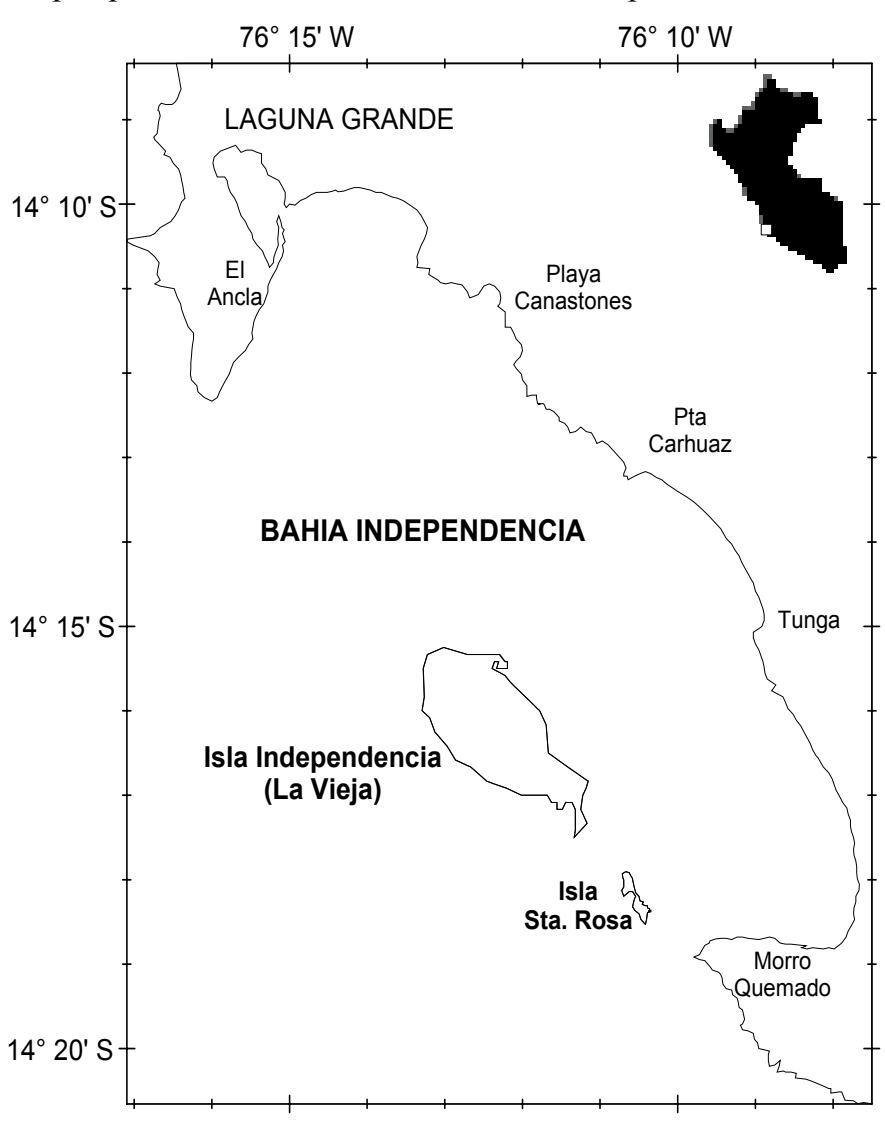

situada al sur de Pisco (Figura 1) y una de las especies comerciales más importantes es la concha de abanico (Argopecten purpuratus) cuyos volúmenes de desembarque se incrementan explosivamente durante eventos El Niño fuertes como el de los años 1983/84 o 1997/98.

En 1983/84, bajo el impacto del fenómeno El Niño, la temperatura superficial del mar en los bancos de concha de abanico de $16^{\circ} \mathrm{C}$ durante un verano normal se incrementaron hasta casi los $25^{\circ} \mathrm{C}$; con ello muchas áreas fueron recolonizadas y la población de concha de abanico se incrementó enormemente de unos cientos de toneladas a cerca de $30000 \mathrm{t}$ en pocos meses (Wolf, 1985; 1987). Estudios realizados sobre la dinámica poblacional de esta especie en años normales y con El Niño (Wolff \& Wolff 1983; Wolff 1985, 1987, Mendo et al., 1988; Mendo \& Yamashiro 1988; Mendo \& Jurado, 1993) han sugerido que el crecimiento poblacional de concha de abanico se debe principalmente a: 1) incremento en la tasa de crecimiento; 2) la reducción del periodo larval y así la mortalidad larval; 3) incremento de la mortalidad natural de predadores; 4) incremento de los productos sexuales y 5) reducción de los competidores filtradores. Sin embargo, solo algunos de estos factores han sido evaluados con información relevante. Así mismo aun cuando se presumía que durante El Niño 1997/98 en términos de producción sería similar al de El Niño 1983/84, los desembarques fueron mucho menores probablemente debido a inapropiadas estrategias de explotación aplicadas por pescadores y empresarios con la consecuente pérdida de biomasa y económica (Wolff \& Mendo, 2000; Mendo \& Wolff, 2002; Skrabonja \& Mendo, 2002; Proleón \& Mendo, 2002)

El presente estudio analiza los cambios de los desembarques de la concha de abanico con relación a los cambios de temperatura en la Bahía Independencia durante las ultimas dos décadas y muestra evidencias de los cambios de algunos factores bióticos y abióticos como crecimiento, reproducción, predación, densidad media y condiciones de oxígeno considerados como causales del incremento de la producción durante los eventos fuertes de El Niño en 1983 y 1998.

Figura 1. Mapa de la Bahía Independencia 


\section{Materiales y métodos}

\section{Algunas características del área de estudio y la pesquería}

La Bahía Independencia (Figura 1) esta localizada a $250 \mathrm{Km}$ al sur de Lima. Tiene una superficie de alrededor de $150 \mathrm{~km}^{2}$, una profundidad promedio de cerca de $25 \mathrm{~m}$ y esta fuertemente influenciada por el núcleo de afloramiento del sur $\left(14^{\circ} \mathrm{S}-16^{\circ} \mathrm{S}\right)$ (Zuta et.al., 1978). La mayor parte del sustrato de la bahía es arenoso o pedregoso, pero existen también áreas con sustrato rocoso en la parte sur de la bahía. Los vientos que soplan de sur a norte, son fuertes (hasta 7 nudos) en las tardes y causan una mezcla constante en la columna de agua. Las temperaturas durante el año fluctúan normalmente entre $12^{\circ} \mathrm{C}$ (fondo) y $16^{\circ} \mathrm{C}$ (superficie) y los niveles de oxígeno se encuentran cerca del nivel de saturación en aguas someras (< $15 \mathrm{~m})$ y bajan drásticamente a valores críticos para invertebrados $\left(<1 \mathrm{ml} \mathrm{l}^{-1}\right)$ a $30 \mathrm{~m}$ de profundidad (Wolff 1985). Durante eventos fuertes de El Niño como el de $1982 / 83$ y $1997 / 98$ las temperaturas alcanzan valores $>20^{\circ} \mathrm{C}$, la transparencia se incrementa a $>10 \mathrm{~m}$ al igual que el oxígeno cerca del fondo $(>6 \mathrm{ml} / \mathrm{l}$ ) (Wolff 1985, Wolff \& Mendo, 2000).

La pesquería de concha de abanico se concentra a una profundidad que oscila entre 5 y $30 \mathrm{~m}$, la cual varía con la zona. La producción diaria de los buzos depende mucho de la visibilidad por lo que solo operan durante las horas con luz (usualmente entre 9:00 y 14:00). La mayoría de los buzos permanecen sumergidos entre 3 y 5 horas al día.

\section{Base de datos usados en el presente estudio}

Los datos de desembarque anual y biomasa de concha de abanico fueron tomados de informes publicados por el Instituto del Mar del Perú (Flores et al., 1994; Estrella \& Guevara, 1998; Mendo et al., 1987; 1989) y de informes no publicados por Mendo \& Wolff). La serie de tiempo de temperatura del mar superficial registrado en base diaria en la Isla La Vieja (Figura 2) fue usada para calcular las anomalías de temperatura mensuales, las cuales fueron relacionadas con los desembarques de concha de abanico. Se asumió que la variación de las capturas podría ser explicada por las anomalías de temperatura tal como lo sugiere Arntz \& Fahrbach (1991) y Wolff (1985). Ya que no se observó una clara relación entre la temperatura y las capturas incluso cuando se trabajo con desfases en las series de tiempo o cuando los datos de temperatura y las capturas fueron promediados a diferentes intervalos de tiempo, se trato de correlacionar la temperatura promedio de los meses de mayor actividad reproductiva de la concha de abanico (Noviembre-Abril) y las capturas acumuladas de los subsiguientes doce meses (Mayo-Abril) bajo el supuesto que la temperatura afecta significativamente la reproducción y el desarrollo y supervivencia larval de la concha de abanico (Wolff 1987, 1988).

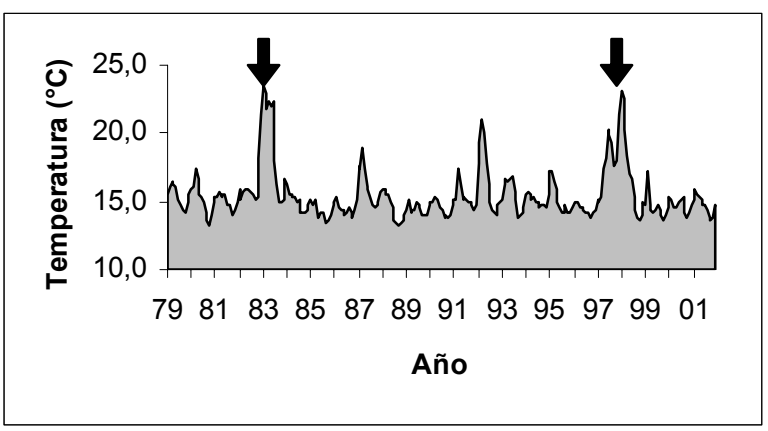

Figura 2. Serie de tiempo mensual de la temperatura superficial del mar en la Bahía Independencia durante 1979 a 2001. Las flechas indican los eventos del El Niño 1983 y 1998.

Finalmente, datos publicados sobre crecimiento de la concha de abanico (Wolf, 1987; Yamashiro \& Mendo, 1988; Mendo \& Jurado, 1993: Mendo, 1999; Porst, 2002; Skrabonja \& Mendo, 2002; Mendo et al, 2002) fueron usados para calcular y relacionar el índice de rendimiento en crecimiento ( $\phi$ ') con la temperatura. El índice de rendimiento en crecimiento fue calculado usando los parámetros de la ecuación de von Bertalanffy K y Loo ( $\phi$ ' $=\log \mathrm{K}+2 \log L o o$ ) (Pauly \& Munro 1984).

Así mismo datos de biomasa de predadores, índice gonadosomático y concentración de oxígeno registrados durante las evaluaciones de la población de concha de abanico (Mendo et al., 1987, 1988, 1989; Yamashiro et al., 1990; Rubio et al., 1998; Samame et al., 1985), fueron revisados y reanalizados con la finalidad de explicar las causales de los cambios en la producción de concha de abanico durante los eventos El Niño.

\section{Resultados y discusión}

Relación entre la temperatura superficial del mar y las capturas de concha de abanico

La Figura 3 muestra las capturas mensuales de concha de abanico y las anomalías mensuales de temperatura superficial del mar durante el periodo 1983 a 2002. Las temperaturas máximas en el periodo se presentan en los años 1983 y 1998 y los mayores volúmenes de captura se presentan aproximadamente un año después de estos máximos de las anomalías de temperatura. También se puede observar un pico en el período 1985/86, sin embargo este no correlaciona con altas temperaturas del año anterior. Asimismo, en el año 1992 las temperaturas fueron $5^{\circ} \mathrm{C}$ por arriba de lo normal, y aun así no se registró un efecto sobre los desembarques. Ello nos permite concluir que en 
muchos casos los niveles altos o bajos de captura de concha de abanico no son explicados por los cambios de temperatura y solo existe una correlación muy alta cuando se trata de periodos con eventos fuertes de El Niño. La Figura 4 muestra esta correlación significativa entre los desembarques de concha de abanico y la temperatura promedio durante el periodo reproductivo de este recurso previo a la temporada de pesca.

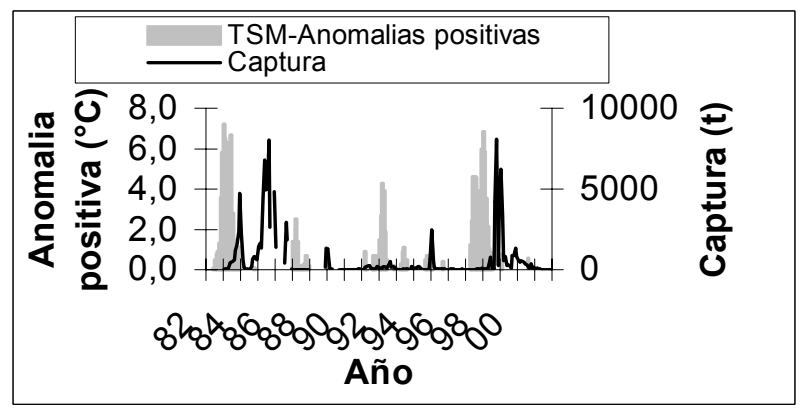

Figura 3. Relación entre las anomalías mensuales de la temperatura superficial del mar $\left({ }^{\circ} \mathrm{C}\right.$, barras $)$ y las capturas de concha de abanico (t, línea) en la Bahía Independencia.

La predicción de las capturas de concha de abanico después del periodo reproductivo basado en la temperatura durante ese periodo tiene limitaciones fuertes, y solo podría ser usado para niveles de impacto fuertes originados durante eventos fuertes de El Niño. Es preciso mencionar que los intentos de correlacionar la temperatura (valores absolutos y anomalías) con las capturas (usando desfases de 3 y 6 meses entre ambos) a nivel mensual, estacional y semestral, no mejoraron los índices de correlación. Esto nos lleva a la siguiente interpretación: 1) se requiere de varios meses de elevada temperatura en el verano para incrementar significativamente la actividad reproductiva y el reclutamiento de la concha de abanico (determinado por el promedio de temperatura durante el periodo reproductivo) y 2) se requiere mas tiempo para que el cohorte incremente su biomasa y pueda ser convertido en capturas. En algunos años esto podría tomar entre 3 y 4 meses y en otros (de bajo reclutamiento y/o crecimiento más lento) podría tomar por lo menos 12 meses. El modelo de regresión entre ambas variables solo explica el 75\% de la variabilidad inherente a los datos. El $25 \%$ de la variabilidad no explicado por el modelo posiblemente se deba a que no se usaron datos de biomasa en lugar de capturas, los cuales no estuvieron disponibles. Así mismo es importante mencionar que los volúmenes de captura no solo dependen de la abundancia de la especie, sino (por lo menos parcialmente) también de su valor en el mercado no tomado en cuenta en este estudio. Ocasionalmente los buzos colectan especies con poca abundancia si los precios son altos.

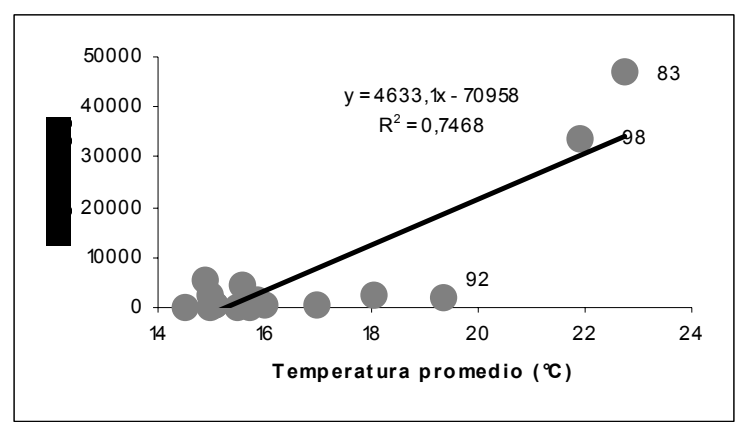

Figura 4. Relación entre la temperatura promedio del mar durante el periodo reproductivo y los subsiguientes desembarques de concha de abanico en la Bahía Independencia.

La naturaleza multifuncional del fenómeno El Niño puede ser una razón mas. Nosotros consideramos solo la temperatura como variable predictiva aun cuando sabemos que otros factores bióticos y abióticos podrían influenciar el desarrollo poblacional de concha de abanico y de otras especies del ecosistema. Las altas concentraciones de oxígeno en los bancos de concha de abanico registradas durante los años El Niño, podrían ser el resultado de vientos fuertes poco usuales que mezclan las aguas de la bahía y de la intrusión de Aguas Subtropicales Superficiales en la Bahía con alta concentración de oxígeno (Com. Pers. O. Morón).

Una conclusión importante es que el incremento de la población de concha de abanico necesita condiciones favorables de temperatura por varios meses tal como en los años 1983 y 1998. Aun cuando en 1992 se registró un periodo de calentamiento este no originó una explosión de la población de concha de abanico en la bahía.

\section{Factores que afectan la producción de concha de abanico durante EI Niño}

La relación entre el índice de rendimiento en crecimiento $(\phi ')$ y la temperatura muestran claramente que la concha de abanico crece mejor bajo condiciones de calentamientos del mar (Figura 5a). Igualmente el índice gonadosomático durante El Niño se incrementa considerablemente (Figura 5b), lo cual sugiere que la actividad reproductiva en general se incrementa en condiciones de mar calientes. Wolff (1988) encontró que el tiempo de recuperación entre dos sucesivos desoves fue significativamente mas corto durante El Niño 1983 indicando que la maduración fue acelerada por las altas temperaturas del mar. También Disalvo et al. (1984) demostró en experimentos de laboratorio que las larvas desarrollaron dos veces mas rápido a $25^{\circ} \mathrm{C}$ que a $19^{\circ} \mathrm{C}$ y con una alta tasa de supervivencia.

Por otro lado la Figura $5 \mathrm{c}$ muestra la relación directa entre la concentración de oxígeno y las densidades de 


\section{JAIME MENDO Y MATTHIAS WOLFF}

Ecología Aplicada Vol. $2 \mathrm{~N}^{\circ}$ 1, pp. 51-57

concha de abanico, lo cual sugiere que las elevadas densidades de concha de abanico durante El Niño se deben por lo menos en parte a los elevados niveles de oxígeno.

Finalmente la biomasa de los principales predadores de concha de abanico en un año con el evento El Niño (1998) es mucho menor que durante un año normal (1988) (Figura 5d), hecho que fue reportado también para El Niño $1983 / 84$ por Wolff (1987).

Nuestra revisión sugiere que el "boom" del stock de concha de abanico es debido al efecto combinado de: (1) incremento en la actividad reproductiva a través de una aceleración de la maduración y un incremento de la frecuencia de desove; (2) acortamiento del período larval e incremento en la supervivencia larval; (3) incremento en el rendimiento en crecimiento individual; (4) incremento en la supervivencia de juveniles y adultos debido a la reducción de la biomasa de predadores; (5) incremento en la capacidad de carga de los bancos de conchas debido a elevados niveles de oxígeno.

Es conocido que el calentamiento de El Niño conduce a acelerar el metabolismo de concha de abanico (crecimiento y reproducción) y por lo tanto una fuente rica en alimento debe estar disponible para sostener el crecimiento y el desarrollo de la población. Sin embargo, durante el evento El Niño 97/98 la producción primaria decrece significativamente, tal como se puede apreciar en la Fig. 6. Dos explicaciones (no necesariamente exclusivas) al respecto pueden ser planteadas: 1) en años normales, el alimento para especies filtradoras se encuentra en exceso y lejos del nivel limitante y permanece así aun si los niveles bajan durante El Niño, 2) la concha de abanico usa una fuente de alimento diferente bajo condiciones de El Niño, posiblemente Bacterias enriquecidas con detritus en suspensión, las cuales periódicamente son suspendidas en la columna de agua por las corrientes de marea. De esta manera Bacterias y Protozoos podrían ser la principal fuente de alimento durante El Niño, una hipótesis que todavía no ha sido probada. En este contexto es interesante notar que durante el evento fuerte de El Niño 1983/84 grandes stocks de langostinos fueron introducidos en la zona de pesca de Pisco por una lengua de agua tropical caliente proveniente del norte de Perú y Ecuador. Tanto los langostinos y la concha de abanico no mostraron signos de escasez de alimento. El alimento definitivamente no fue un factor limitante a pesar de la reducción de la producción primaria.

Al discutir los factores que afectan el desarrollo de la población de concha de abanico durante un evento fuerte de El Niño, es importante considerar a la pesquería en detalle. Tal como se mencionó antes, los volúmenes de captura no solo dependen de la abundancia relativa de la especie sino también del valor del mercado y obviamente del esfuerzo pesquero y la estrategia de pesca que se le aplica. Si la pesquería permite crecer a la concha de abanico hasta un tamaño adulto $(>65 \mathrm{~mm})$ respetando así la talla mínima legal de captura, el cohorte anual entonces estaría en la capacidad de formar su biomasa y de desovar varias veces. De esta manera los procesos reproductivos y los reclutamientos serian importantes y más intensos que en años normales y el incremento del stock se podría prolongar por más tiempo. Este fue el caso durante el evento El Niño 1983/84, cuando la concha de abanico de Bahía Independencia no fue explotada hasta Agosto de 1983, cuando la mayoría de las conchas ya habían crecido a tamaños $>70 \mathrm{~mm}$ (Wolff, 1987). A partir de entonces, aun cuando la biomasa fue reducida fuertemente por la pesquería, continuaron los pulsos fuertes de la población en los años 1984 y 1985 (Figura 3) debido a la existencia de una biomasa desovante alta en el cohorte original de 1983. Sin embargo, esta situación no duro mucho y luego mayormente debido a la implementación de una línea de exportación a fines de 1983, la presión pesquera se incremento en muchos casos sin restricciones ni cuotas de captura. Durante el ultimo evento fuerte de El Niño en 1998 la figura fue diferente a 1983: la concha de abanico fue colectada tan pronto aparecían los nuevos reclutas en los bancos y el esfuerzo de pesca aplicado fue enorme (Wolff \& Mendo, 2000; Mendo \& Wolff, 2002). Como un resultado de esta sobreexplotación por crecimiento, la biomasa del stock no se incremento como durante 1983 a pesar de las altas densidades de reclutas existentes $\left(>300 / \mathrm{m}^{2}\right)$ en Junio de1998 (Mendo \& Wolff, observaciones de buceo).

La experiencia acumulada y el análisis realizado nos permite concluir que el incremento de la producción anual de la población de concha de abanico en Bahía Independencia depende de la ocurrencia de eventos fuertes de El Niño y que el rendimiento total obtenido depende fuertemente de la estrategia de pesca, es decir, de la talla de primera captura, la cual debería ser mayor de $65 \mathrm{~mm}$ de altura. Mendo \& Wolff (2002) han planteado algunas medidas de manejo adicionales que deberían tomarse en cuenta en el caso de una nueva aparición de un evento fuerte de El Niño.

\section{Agradecimientos}

Esta publicación fue concluida durante una pasantía del primer autor en el Centro de Ecología Marina Tropical (ZMT) de la Universidad de Bremen. Nuestro agradecimiento a aquellas personas e instituciones que proporcionaron alguna información relevante para este estudio. 

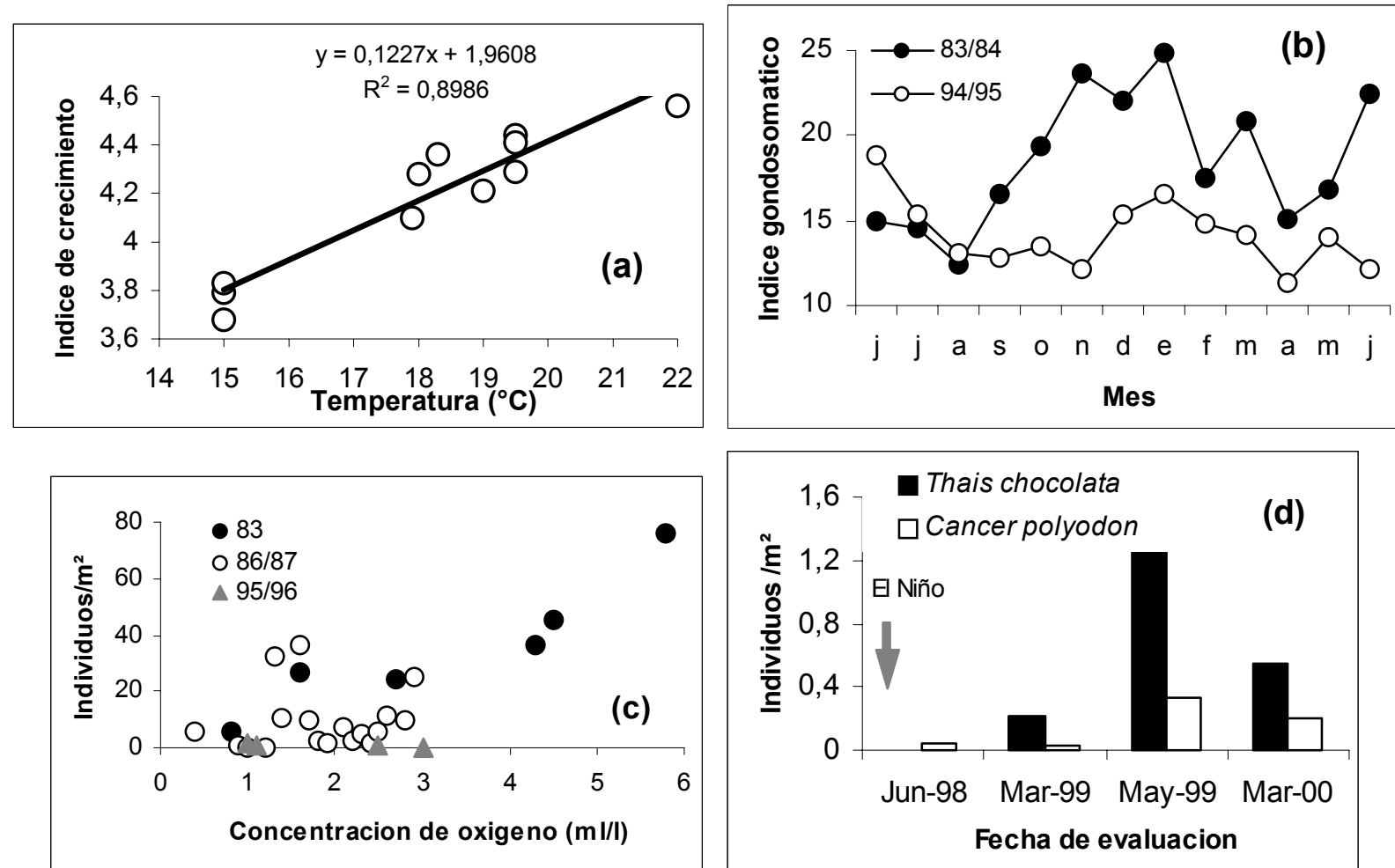

Figura 5. (a) Relación entre el índice de rendimiento en crecimiento ( $\left.\phi^{\prime}\right)$ de la concha de abanico y la temperatura del mar $\left({ }^{\circ} \mathrm{C}\right)$; (b) Índice gonadosomático durante El Niño y condiciones normales; (c) Concentración de oxigeno $(\mathrm{ml} / \mathrm{l})$ versus densidad $\left(\mathrm{n} / \mathrm{m}^{2}\right)$ de concha de abanico; (d) Densidad $\left(\mathrm{n} / \mathrm{m}^{2}\right)$ de predadores de concha de abanico durante El Niño y condiciones normales en la Bahía Independencia.

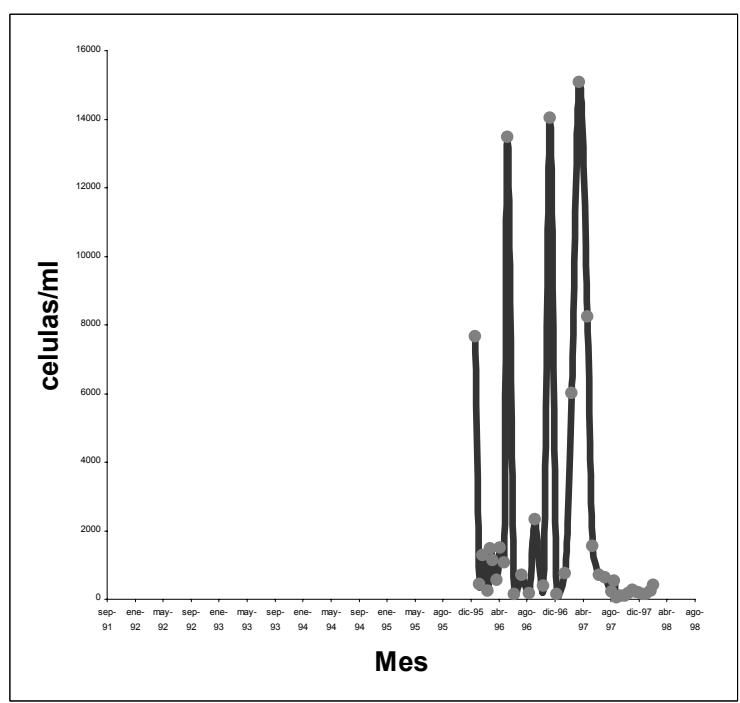

Figura 6. Número de células de fitoplancton $(\mathrm{n} / \mathrm{ml})$ registradas en Bahía Independencia durante 1995 a 1998 (Datos proporcionados por Noemí Ochoa)

\section{Literatura citada}

Arntz W. \& Fahrbach E. 1991. El NiñoKlimaexperiment der Natur. Berlin, Birkhäuser Verlag.

DiSalvo L.H., Alarcón E., Martinez E. \& Uribe E. 1984. Progress in mass culture of Argopecten purpuratus (Lamarck 1819) with notes on its natural history. Rev.Chilena de Hist.Nat. 57: 33-45

Espino M. \& Wosnitza-Mendo C. 1988. La pesquería artesanal y la variabilidad de los recursos en el Perú. p. 121-144. En C. Wosnitza-Mendo, M. Espino y M. Veliz (eds.) La Pesquería Artesanal en el Perú durante Junio de 1986 a Junio de 1988. Inf. Inst. Mar del Perú. 93.

Estrella C. \& Guevara R. 1998. Informe estadístico anual de los recursos hidrobiológicos de la pesca artesanal por especies, artes, caletas y meses durante 1997. Inf. Inst. Mar del Perú. 132.

Flores M.,Vera S., Marcelo R. \& Chirinos E. 1994. Estadísticas de los desembarques de la pesquería marina Peruana 1983-1992. Inf. Inst. Mar del Perú. 105.

Mendo J. 1999. Effect of some environmental variables on age and growth of the Peruvian scallop (Argopecten purpuratus) in suspended and bottom cultures, Independencia Bay, Pisco, Perú. Scientific Report. International Foundation for Science. 
Mendo J. \& Jurado E. 1993. Length-based growth parameter estimates of the Peruvian scallop (Argopecten purpuratus). Fisheries Research. 15: 357-367.

Mendo J., Yamashiro C., Rubio J., Kameya A., Jurado E., Maldonado M. \& Guzmán S. 1989. Evaluación de la población de concha de abanico (Argopecten purpuratus) en la Bahía Independencia, Pisco, Perú (23 de Setiembre-9 de Octubre de 1987). Inf. Inst. Mar Perú-Callao. (94).

Mendo J., Valdivieso V., Yamashiro C., Jurado E., Morón O. \& Rubio J. 1987. Evaluación de la población de concha de abanico (Argopecten purpuratus) en la Bahía Independencia, Pisco, Perú (17 de Enero-4 de Febrero de 1987). Inf. Inst. Mar Perú-Callao. (91).

Mendo J., Valdivieso V. \& Yamashiro C. 1988. Cambios en densidad, numero y biomasa de la concha de abanico (Argopecten purpuratus) en la Bahía Independencia (Pisco, Perú) durante 19841987. pp. 163-168. En: Salzwedel, H. y A. Landa. (eds.). Recursos y dinámica del ecosistema de afloramiento Peruano. Bol. Inst. Mar Perú-Callao, Vol. extr.

Mendo J. \& Wolf M. 2002. Pesquería y manejo de la concha de abanico (Argopecten purpuratus) en la Bahía Independencia. En: J. Mendo y M. Wolf (eds) Memorias de la I Jornada Científica de la Reserva Nacional de Paracas, 28-31 Marzo Pisco. del 2001. Univ. Nac. Agraria La Molina.

Pauly D. \& Munro J.L. 1984. Once more on the comparison of growth in fish and invertebrates. Fishbyte. 2(1): 21.

Proleon J. \& Mendo J. 2002. Estrategia adoptada por los pescadores artesanales ante el reclutamiento masivo de concha de abanico (Argopecten purpuratus) en la Bahía Independencia, 1997-2000 Pisco. En: J. Mendo y M. Wolf (eds) Memorias de la I Jornada Científica de la Reserva Nacional de Paracas, 28-31 Marzo Pisco. del 2001. Univ. Nac. Agraria La Molina.

Rubio J., Taipe A. \& Aguilar S. 1998. Evaluación de la concha de abanico (Argopecten purpuratus) en Bahía Independencia, 15-27 de Julio 1998. Resumen Ejecutivo, Inst. Mar Perú - Callao.

Ryther J.H. 1969. Photosynthesis and fish production in the sea. Science. 166: 72-76.

Samame M., Benites C., Valdivieso V., Mendez M., Yamashiro C. \& Morón O. 1985. Evaluación del recurso concha de abanico (Argopecten purpuratus) en la Bahía Independencia y otros bancos naturales de la provincia de Pisco, en mayo 1985. Informe interno Inst. Mar Perú.

Skrabonja A. \& Mendo J. 2002. Impacto de la actividad de repoblamiento, sobre el rendimiento biológico de la concha de abanico (Argopecten purpuratus) en Bahía Independencia En: J. Mendo y M. Wolf (eds) Memorias de la I Jornada Científica de la Reserva Nacional de Paracas, 2831 Marzo Pisco. del 2001. Univ. Nac. Agraria La Molina.

Wolff M. \& Wolff R. 1983. Observations on the utilization and growth of the pectinid Argopecten purpuratus in the fishing area of Pisco, Perú. Bol. Inst. Mar Peru. 7(6): 197-235.

Wolff M. 1985. Abundancia masiva y crecimiento de pre-adultos de la concha de abanico Peruana (Argopecten purpuratus) en la zona de Pisco bajo condiciones de "El Niño" 1983. pp. 87-90. En: Arntz, W., A. Landa y J. Tarazona (eds.). "El Niño" y su impacto en la fauna marina. Bol. Inst. Mar Perú-Callao, Vol. extr.

Wolff M. 1987. Population Dynamics of the Peruvian scallop Argopecten purpuratus during the El Niño Phenomenon of 1983. Can. J. Fish. Aquat. SCI. 44: 1684-1691.

Wolff M. 1988. Spawning and recruitment in the Peruvian scallop Argopecten purpuratus. Mar. Ecol. Prog. Ser. 42: 213-217.

Wolff M. \& Mendo J. 2000. Management of the Peruvian Bay Scallop (Argopecten purpuratus) metapopulation with regard to environmental change. Aquatic Conserv: Mar. Freshw. Ecosyst. 10: 117-126.

Yamashiro C., Rubio J., Jurado E., Maldonado M., Auza E., Ayon P. \& Antonieti E. 1990. Evaluación de la población de concha de abanico, Argopecten purpuratus, en la Bahía Independencia, Pisco, Perú. Inst. Mar. Perú. Inf. Nro. 48.

Yamashiro C. \& Mendo J. 1988. Crecimiento de la concha de abanico (Argopecten purpuratus) en la Bahía Independencia, Pisco, Perú. pp. 163-168. En: Salzwedel H. y A. Landa (eds.) Recursos y dinámica del ecosistema de afloramiento peruano. Bol. Inst. Mar Perú-Callao, Vol. extr.

Zuta S., Rivera T. \& Bustamante A. 1978. Hydrologic aspects of the main upwelling areas off Perú. P.235-257. In R. Boje and M. Tomczak. Upwelling Ecosystems. Springer-Verlag, Berlin. 